\title{
BCS-BEC crossover in two dimensions: a Quantum Monte Carlo study
}

\author{
G. Bertaina \\ Institute of Theoretical Physics, Ecole Polytechnique Fédérale de Lausanne EPFL, CH-1015 \\ Lausanne, Switzerland
}

\begin{abstract}
We investigate the crossover from Bardeen-Cooper-Schrieffer (BCS) superfluidity to Bose-Einstein condensation (BEC) in a two-dimensional Fermi gas at $T=0$ using the fixed-node diffusion Monte Carlo method. We calculate the equation of state and the gap parameter as a function of the interaction strength, observing large deviations compared to mean-field predictions. In the BEC regime our results show the important role of dimer-dimer and atom-dimer interaction effects that are completely neglected in the mean-field picture. We also consider the highly polarized gas and the competition between a polaronic and a molecular picture.
\end{abstract}

Keywords: BCS-BEC crossover, Diffusion Monte Carlo, low dimensionality

PACS: 05.30.Fk, 03.75.Hh, 03.75.Ss

The study of ultracold atomic Fermi gases has become an active and rich field of research [1]. Important areas of investigation include the BCS-BEC crossover in a superfluid gas with resonantly enhanced interactions, the Chandrasekhar-Clogston instability of the superfluid state when spin polarization is increased, the possible onset of itinerant ferromagnetism in a gas with repulsive interactions [2] and the realization of the Hubbard model for fermions loaded in optical lattices [1].

Low dimensional configurations of degenerate Fermi gases have also been the object of experimental and theoretical studies [1]. In particular, a two-dimensional (2D) ultracold Fermi gas has been recently realized using a highly anisotropic pancake-shaped potential, and the density profile of the cloud has been measured using in situ imaging [3]; moreover the single particle spectral function has been measured by means of rf spectroscopy [4]. On the theoretical side, the evolution from a superfluid state with large Cooper pairs to one with tight molecules in a 2D system of attractive fermions was first investigated by Miyake [5] and later by Randeria and coworkers [6] aiming to describe high- $T_{c}$ superconductors. More recent studies are in general based on perturbative or mean-field (MF) approaches that are suitable in the regime of weak coupling, but are bound to break down for stronger interactions (see references in [8]). Recently we have obtained the first determination using quantum Monte Carlo methods of the equation of state at $T=0$ of a homogeneous 2D Fermi gas in the BCS-BEC crossover [8].

In this paper we review the main results that we have more extensively shown in [8], concerning the equation of state and the pairing gap as a function of the interaction strength. We show that in the strong-coupling regime the emergence of interaction effects involving dimers produce large deviations compared to MF predictions. We also discuss the problem of the polaron-molecule transition in a two-dimensional Fermi gas. 
Method. We consider a homogeneous two-component Fermi gas described by the Hamiltonian

$$
H=-\frac{\hbar^{2}}{2 m}\left(\sum_{i=1}^{N_{\uparrow}} \nabla_{i}^{2}+\sum_{i^{\prime}=1}^{N_{\downarrow}} \nabla_{i^{\prime}}^{2}\right)+\sum_{i, i^{\prime}} V\left(r_{i i^{\prime}}\right),
$$

where $m$ denotes the mass of the particles, $i, j, \ldots$ and $i^{\prime}, j^{\prime}, \ldots$ label, respectively, spinup and spin-down particles and $N_{\uparrow}=N_{\downarrow}=N / 2, N$ being the total number of atoms. We model the interspecies interatomic interactions using an attractive square-well (SW) potential: $V(r)=-V_{0}$ for $r<R\left(V_{0}>0\right)$, and $V(r)=0$ otherwise. In order to ensure that the mean interparticle distance is much larger than the range of the potential we use $n R^{2}=10^{-6}$, where $n$ is the gas number density, or equivalently $k_{F} R=0.0025$ in terms of the Fermi wave vector $k_{F}=\sqrt{2 \pi n}$. We simulate a strictly $2 \mathrm{D}$ system and describe the low-energy collisions of the SW potential in terms of the $2 \mathrm{D}$ scattering length $a_{2 D}$ defined as $a_{2 D}=R e^{J_{0}(\kappa) / \kappa J_{1}(\kappa)}$, where $J_{0(1)}(x)$ are Bessel functions of the first kind and $\kappa=\sqrt{V_{0} m R^{2} / \hbar^{2}}$. The scattering length is non negative and diverges at $\kappa=0$ and at the zeros of $J_{1}$, corresponding to the appearance of new two-body bound states in the well. Close to these points the shallow dimers have size $a_{2 D}$ and their binding energy is given by $\varepsilon_{b}=-4 \hbar^{2} /\left(m a_{2 D}^{2} e^{2 \gamma}\right)$, where $\gamma \simeq 0.577$ is Euler's constant. The dependence of $a_{2 D}$ on the depth $V_{0}$ in the region where the well supports only one bound state is shown in the inset of Fig. 1. Two regions are clearly identified by comparing $a_{2 D}$ with the mean interparticle distance $1 / k_{F}$ : i) $k_{F} a_{2 D} \gg 1$ corresponds to the BCS regime where interactions are weak and dimers are large and weakly bound, ii) $k_{F} a_{2 D} \ll 1$ corresponds to the BEC regime of tightly bound composite bosons. Compared to the $3 \mathrm{D}$ case the BCS-BEC crossover in 2D exhibits important differences. a) For a purely attractive potential a two-body bound state exists for arbitrarily weak attractions. b) The weak-coupling limit corresponds to a diverging scattering length $a_{2 D}$. c) The 2D scattering amplitude of particles colliding at low energy is given by $f(k)=2 \pi /\left[\log \left(2 / k a_{2 D} e^{\gamma}\right)+i \pi / 2\right]$ [7]. There is no range of values of $a_{2 D}$ for which $f(k)$ is independent of interaction (unitary limit). d) The mean-field coupling constant can be written as $g=\left(2 \pi \hbar^{2} / m\right) / \log \left(1 / k_{F} a_{2 D}\right)$ with logarithmic accuracy. Within the same accuracy, the region $k_{F} a_{2 D} \sim 1$ identifies the strong-coupling crossover between the BCS and the BEC regimes [see inset of Fig. 1].

Simulations are carried out using the fixed-node diffusion Monte Carlo (FN-DMC) method. This numerical technique yields an upper bound for the ground-state energy of the gas, resulting from an ansatz for the nodal surface of the many-body wave function that is kept fixed during the calculation (see Refs. [9, 8] for more details). The boundary condition is enforced using a trial function that we choose of the general form $\psi_{T}(\mathbf{R})=\Phi_{S}(\mathbf{R}) \Phi_{A}(\mathbf{R}) . \Phi_{S}$ is a positive function of the particle coordinates and is symmetric in the exchange of particles with equal spin, while $\Phi_{A}$ satisfies the fermionic antisymmetry condition and determines the nodal surface of $\psi_{T}$. The symmetric part is chosen of the Jastrow form $\Phi_{S}(\mathbf{R})=\prod_{i, i^{\prime}} f_{\uparrow \downarrow}\left(r_{i, i^{\prime}}\right) \prod_{i<j} f_{\uparrow \uparrow}\left(r_{i j}\right) \prod_{i^{\prime}<j^{\prime}} f_{\downarrow \downarrow}\left(r_{i^{\prime} j^{\prime}}\right)$, where two-body correlation functions of the interparticle distance have been introduced. Two important regimes are described by the $\Phi_{A}$ component: i) if it is an antisymmetrized product $\Phi_{A}(\mathbf{R})=\mathscr{A}\left(\phi\left(r_{11^{\prime}}\right) \phi\left(r_{22^{\prime}}\right) \ldots \phi\left(r_{N_{\uparrow} N_{\downarrow}}\right)\right)$ of pairwise orbitals $\phi$ corresponding to the two-body bound state of the potential $V(r), \psi_{T}(\mathbf{R})$ describes a BCS state of 


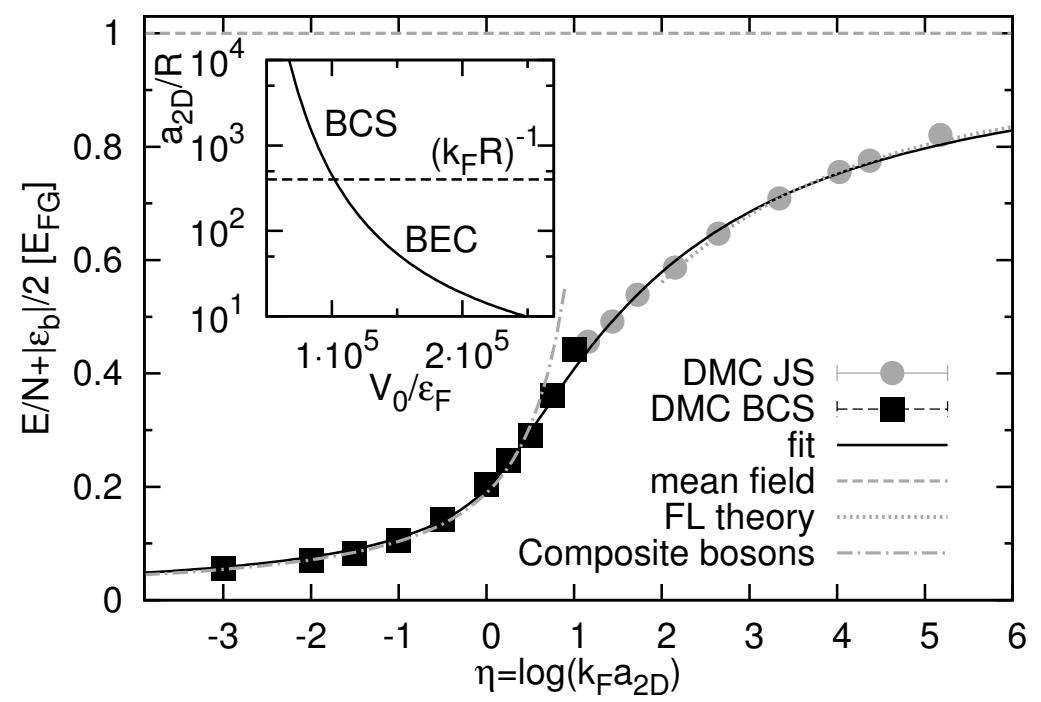

FIGURE 1. Equation of state in the BCS-BEC crossover with $\varepsilon_{b} / 2$ subtracted from $E / N$. Squares refer to the BCS and circles to the JS wave function. The solid line is a fit to the data, the dot-dashed line shows the equation of state (2) of composite bosons and the dotted line shows the perturbation expansion holding in the BCS regime (see text). The dashed line is the MF result. Inset: 2D scattering length $a_{2 D}$ as a function of the depth $V_{0}$ for a SW potential of radius $R$. The BCS and BEC regimes correspond, respectively, to $k_{F} a_{2 D} \gg 1$ and $k_{F} a_{2 D} \ll 1$.

dimers that is expected to be appropriate in the deep BEC regime; ii) if instead $\Phi_{A}(\mathbf{R})=$ $D_{\uparrow}\left(N_{\uparrow}\right) D_{\downarrow}\left(N_{\downarrow}\right)$, namely the product of the plane-wave Slater determinants for spin-up and spin-down particles, $\psi_{T}$ is a typical Jastrow-Slater (JS) function of a normal Fermi liquid. This description is expected to hold in the BCS regime of a weakly interacting gas where the effect of pairing on the ground-state energy is negligible.

Energy per particle. In Fig. 1 we show the FN-DMC results for the equation of state as a function of the interaction parameter in units of the energy per particle of the noninteracting gas $E_{F G}=\hbar^{2} k_{F}^{2} / 4 m=\varepsilon_{F} / 2$, where $\varepsilon_{F}$ is the Fermi energy, with $\varepsilon_{b} / 2$ subtracted. The BCS wave function provides a lower energy for values of the interaction parameter $\eta=\log \left(k_{F} a_{2 D}\right) \lesssim 1$, while the JS function is more favorable for larger values of $\eta$. The role of finite-size effects has been investigated using the method described in [10]. In the deep BEC regime, besides the molecular contribution, the remaining fraction of energy corresponds to the interaction energy of the dimers. Concerning this contribution to the energy, the MF result $E / N=E_{F G}+\varepsilon_{b} / 2[5,6]$, which is shown for comparison, misses the expected logarithmic dependence on density and interaction strength. Effects beyond mean-field are therefore much more pronounced in 2D than in 3D (see [1]). In the BEC regime the FN-DMC results are fitted with the equation of state of a gas of composite bosons corresponding to hard disks of diameter $a_{d}$

$$
\frac{E}{N_{d}}+\left|\varepsilon_{b}\right|=\frac{2 \pi \hbar^{2} n_{d}}{m_{d}} \frac{1}{\log \left(1 / n_{d} a_{d}^{2}\right)}\left[1-\frac{\log \log \left(1 / n_{d} a_{d}^{2}\right)}{\log \left(1 / n_{d} a_{d}^{2}\right)}+\frac{\log \pi+2 \gamma+1 / 2}{\log \left(1 / n_{d} a_{d}^{2}\right)}\right]
$$


where $m_{d}=2 m$ is the mass of the dimer, while the number of dimers, and correspondingly their density $n_{d}$, is half of the total number of fermions $N_{d}=N / 2$. The above expression includes beyond mean-field terms [11] and allows for a precise determination of the dimer-dimer scattering length $a_{d}$. We obtain $a_{d}=0.55(4) a_{2 D}$, in agreement with the four-body calculation in Ref. [7]. In the opposite BCS regime, where the contribution of the pairing gap can be neglected, the fermionic equation of state can be described in terms of an attractive normal Fermi liquid (FL). Beyond logarithmic accuracy one has the second-order expansion in terms of $\eta \frac{E}{N}=E_{F G}\left(1-\frac{1}{\eta}+\frac{A}{\eta^{2}}\right)$ [12]. From a best fit we find the result $A=0.06(2)$ for the coefficient of the second-order term. The derivative of the energy density with respect to the coupling constant can be related to the contact parameter $C$ [14], which we have extracted from our Monte Carlo data (see [8]).

Pairing gap. In Fig. 2 we show the results for the pairing gap $\Delta_{\text {gap }}$ in the strongcoupling regime. This quantity is defined from the difference of ground-state energy $E\left(N_{\uparrow}, N_{\downarrow}\right)$ of systems having one and two more (less) particles $\Delta_{\text {gap }}=1 / 2[2 E(N / 2 \pm$ $1, N / 2)-E(N / 2 \pm 1, N / 2 \pm 1)-E(N / 2, N / 2)]$ [13]. At the MF level [5,6] the pairing gap coincides with the result for the order parameter $\Delta_{\text {gap }}=\Delta=\sqrt{2 \varepsilon_{F}\left|\varepsilon_{b}\right|}$ if $\left|\varepsilon_{b}\right|<2 \varepsilon_{F}$, and is given by $\Delta_{\text {gap }}=\varepsilon_{F}+\left|\varepsilon_{b}\right| / 2$ for larger values of $\left|\varepsilon_{b}\right|$. In the BEC regime the quantity $\Delta_{\text {gap }}-\left|\varepsilon_{b}\right| / 2$, shown in the inset of Fig. 2, displays the repulsive interaction effects between unpaired fermionic atoms and bosonic dimers. In fact, the energy of the system with one extra spin-up particle can be written as the sum of the contribution (2) of $N / 2$ dimers and the Fermi-Bose interaction energy $E(N / 2+1, N / 2)=E(N / 2, N / 2)+$ $g_{B F} n_{d}$, where $g_{B F}=3 \pi \hbar^{2} /\left[m \log \left(1 / n_{d} a_{a d}^{2}\right)\right]$ is the coupling constant fixed by the atomdimer reduced mass $2 m / 3$ and the effective scattering length $a_{a d}$. By using the definition of $\Delta_{\text {gap }}$ and the value $a_{d}=0.55 a_{2 D}$ for the dimer-dimer scattering length in the energy functional (2), we find $a_{a d}=1.7(1) a_{2 D}$ from the fit shown in the inset of Fig. 2 . Experimental results concerning the pairing gap [4] have not revealed beyond mean field effects in the pairing gap at finite temperature; it is still not clear whether this is due to temperature or other effects.

Polaron-molecule transition. In order to investigate more on the nature of the correlations between unpaired fermionic atoms and bosonic dimers or single fermionic impurities (polarons) in two dimensions, we have also considered a system consisting of $N_{\uparrow}$ fermions and a single $\downarrow$ fermion, with the same attractive interaction as described above. This problem has been studied variationally in $[16,15]$ and with diagrammatic calculations in ladder approximation in $[17,18]$. We have used the JS and the polarized BCS [13] nodal surfaces in order to study the polaron and the molecular states, respectively. Finite size effects have been corrected by performing simulations with $N_{\uparrow}=13,25,37,49$ and $N_{\uparrow}=14,26,38,50$ with the JS and the polarized BCS wavefunctions, respectively. Preliminary FN-DMC results show that a polaron to molecule transition happens around $\left|\varepsilon_{b}\right| / \varepsilon_{F} \sim 15(1)$.

Acknowledgments. We want to acknowkedge the support of the University of Trento and the INO-CNR BEC Center in Trento, where the main part of the results have been obtained, and the fruitful discussions with S. Giorgini. 


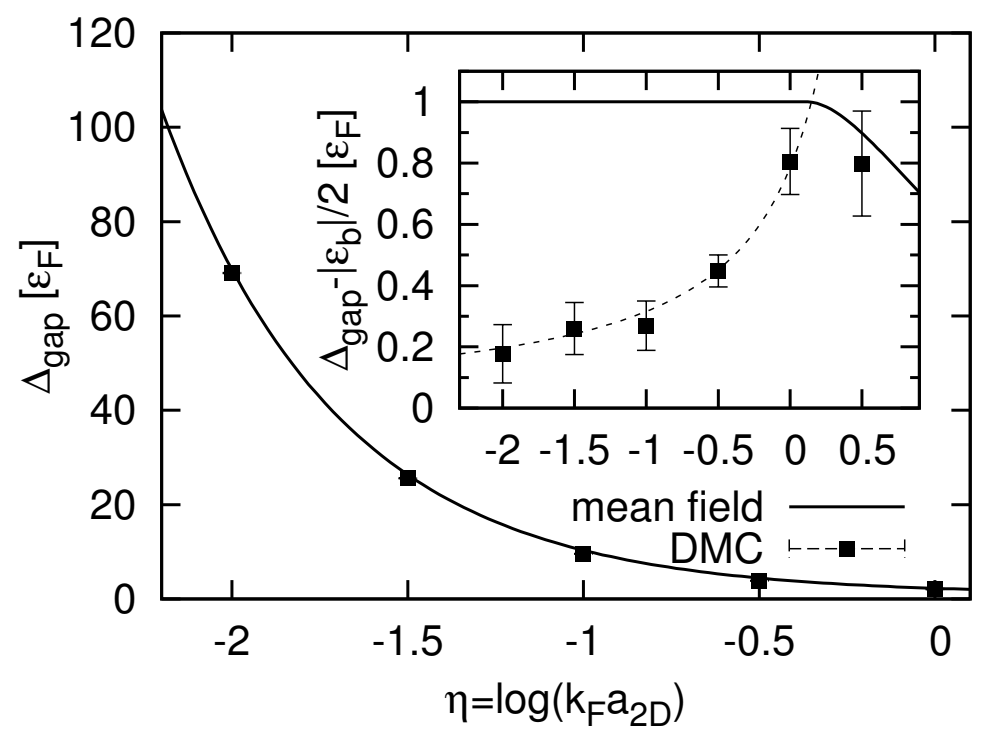

FIGURE 2. Excitation gap in the BCS-BEC crossover. The solid line is the MF result. Inset: excitation gap with $\left|\varepsilon_{b}\right| / 2$ subtracted from $\Delta_{\text {gap }}$. The dashed line is a fit using the energy functional of a Fermi-Bose mixture.

\section{REFERENCES}

1. S. Giorgini, L.P. Pitaevskii and S. Stringari, Rev. Mod. Phys. 80, 1215 (2008); I. Bloch, J. Dalibard and W. Zwerger, Rev. Mod. Phys. 80, 885 (2008).

2. G.-B. Jo et al., Science 325, 1521 (2009).

3. K. Martiyanov, V. Makhalov and A. Turlapov, Phys. Rev. Lett. 105, 030404 (2010); A. Orel et al., New J. Phys. 13, 113032 (2011).

4. B. Fröhlich et al., Phys. Rev. Lett. 106, 105301 (2011); M. Feld, et al., Nature 480, 75 (2011); A. Sommer et al., Phys. Rev. Lett. 108, 045302 (2012).

5. K. Miyake, Prog. Theor. Phys. 69, 1794 (1983).

6. M. Randeria, J.-M. Duan and L.-Y. Shieh, Phys. Rev. Lett. 62, 981 (1989); Phys. Rev. B 41, 327 (1990).

7. D.S. Petrov, M.A. Baranov and G.V. Shlyapnikov, Phys. Rev. A 67, 031601(R) (2003).

8. G. Bertaina and S. Giorgini, Phys. Rev. Lett. 106, 110403 (2011).

9. P.J. Reynolds et al., J. Chem. Phys. 77, 5593 (1982); G.E. Astrakharchik et al., Phys. Rev. Lett. 93, 200404 (2004); S.-Y. Chang et al., Phys. Rev. A 70, 043602 (2004); G.E. Astrakharchik et al., Phys. Rev. Lett. 95, 230405 (2005).

10. C. Lin, F.H. Zong and D.M. Ceperley, Phys. Rev. E 64, 016702 (2001).

11. G.E. Astrakharchik et al., Phys. Rev. A 79, 051602(R) (2009); C. Mora and Y. Castin, Phys. Rev. Lett. 102, 180404 (2009); S.R. Beane, Phys. Rev. A 82, 063610 (2010).

12. P. Bloom, Phys. Rev. B 12, 125 (1975); J.R. Engelbrecht, M. Randeria and L. Zhang, Phys. Rev. B 45, 10135 (1992); J.R. Engelbrecht and M. Randeria, Phys. Rev. B 45, 12419 (1992).

13. J. Carlson et al., Phys. Rev. Lett. 91, 050401 (2003).

14. S. Tan, Ann. Phys. 323, 2952 (2008) and ibid. 2971 (2008).

15. M. Parish, Phys. Rev. A 83, 051603 (2011).

16. S. Zöllner, G. Bruun, and C. Pethick, Phys. Rev. A 83, 021603 (2011).

17. M. Klawunn and A. Recati, Phys. Rev. A 84, 033607 (2011)

18. R. Schmidt et al., arxiv 1110.1649, (2011). 